\title{
Propiedades catalíticas de grafeno dopado con metales de transición
}

\author{
Víctor Aramburu ${ }^{1}$, María López ${ }^{1}$, \\ Héctor Fasoli ${ }^{2}$
}

\footnotetext{
${ }^{1}$ Centro de Investigaciones Fisicoquímicas, Teóricas y Aplicadas (CIFTA), Universidad Nacional de Catamarca, Av. Belgrano 300, 4700, Catamarca, Argentina. e-mail: vmaramburu@gmail.com

${ }^{2}$ Convenio Facultad de Ciencias Exactas y Naturales, Universidad Nacional de Catamarca y Facultad de Ingeniería y Ciencias Agrarias, Pontificia Universidad Católica, Catamarca, Argentina.
}

\section{RESUMEN}

En este trabajo se realiza un estudio basado en la Teoría del Funcional de la Densidad de láminas de grafeno dopadas con Fe, Co y Ni. Se adopta el funcional PBE1PBE y los pseudopotenciales LANL2DZ para los átomos metálicos y las bases 6-31G++(d) para los átomos de carbono. Se analizan tres sistemas formados por la sustitución de un átomo carbono por uno de $\mathrm{Fe}$, $\mathrm{Co}$ y $\mathrm{Ni}$, respectivamente. Se estudiaron las propiedades energéticas, electrónicas y la reactividad química, haciendo una comparación sistemática con una lámina de grafeno puro. Para simular la presencia del solvente en los sistemas de estudio se empleó el modelo SCRF=PCM (Self-Consistent Reaction Field=Polarizable Continuum Model). Asimismo, se determinaron las propiedades catalíticas de los sistemas frente a la adsorción de $\mathrm{O}_{2}$.

Los resultados indican que la presencia del heteroátomos metálicos y agua como solvente modifica la reactividad química y la adsorción de dioxígeno, lo que podría ser determinante en las propiedades catalíticas del grafeno dopado. La lámina de grafeno dopado con níquel resulta la más reactiva y la que presenta energía más favorable frente a la adsorción de oxígeno.

Palabras clave: Grafeno dopado, Catalizador, Adsorción de oxígeno, DFT

\section{ABSTRACT}

In this work a study based on the Density Functional Theory of graphene sheets doped with atoms of Fe, Co and $\mathrm{Ni}$ is carried out. The functional PBE1PBE and the pseudopotentials LANL2DZ for the metal atoms, and the $6-31 \mathrm{G}++(\mathrm{d})$ bases for the carbon atoms were adopted. Three systems formed by the substitution of a carbon atom by one of $\mathrm{Fe}, \mathrm{Co}$ and $\mathrm{Ni}$, respectively, are analyzed. The energetic, electronic and chemical reactivity properties were studied, making a systematic comparison with a pure graphene sheet.

In order to simulate the presence of the solvent in the systems under study, the SCRF = PCM (Self Consistent Reaction Field = Polarizable Continuum Model) was used. In addition, for each system the catalytic properties for $\mathrm{O}_{2}$ adsorption were determined. Our results indicate that the presence of metal heteroatoms and water as solvent modifies the chemical reactivity and the adsorption of dioxygen, which could be determinant on the catalytic properties of doped graphene. The nickel-doped graphene sheet results to be the most reactive and it is the one with the most favorable energy for the adsorption of oxygen.

Keywords: Graphene doped, Catalyst, Adsorption of oxygen, DFT

\section{INTRODUCCIÓN}

El grafeno es una lámina de un átomo de espesor constituida por una disposición hexagonal de átomos de carbono con hibridación sp2. Por sus propiedades únicas, tales como elevada área superficial específica, movilidad de electrones de alta velocidad a temperatura ambiente, excelente conductividad térmica y extraordinarias propiedades mecánicas [1,2,3], ha atraído la atención de diferentes áreas de investigación, en particular la electrocatálisis.

El desarrollo de electrocatalizadores es crucial para las aplicaciones de energías renovables. Se han explorado materiales híbridos como grafeno dopado con metales nobles para este propósito, sin embargo, estos metales están limitados por su escasa disponibilidad y el elevado costo, $[4,5,6]$. En este contexto, los 
metales de transición pueden servir como una alternativa prometedora $[7,8,9]$.

En la búsqueda de nuevos materiales catalíticos los métodos computacionales de primeros principios han demostrado ser útiles herramientas en el diseño racional de catalizadores ya que permiten llegar a resultados y tendencias comparables con datos experimentales, de un modo relativamente rápido, [10].

En este trabajo, se realiza un estudio, utilizando métodos basados en la teoría del funcional de la densidad, de la viabilidad del grafeno con impurezas como potenciales cátodos de celdas de combustible. Se estudiaron tres láminas de grafeno dopadas con $\mathrm{Fe}, \mathrm{Co}$ y $\mathrm{Ni}$, respectivamente, y se analizaron sus propiedades energéticas, electrónicas y reactividad química, realizando una comparación sistemática con una lámina de grafeno puro. Se analiza la influencia de un solvente, utilizando modelo implícito que considera al solvente como un medio continuo. Además, se determinaron las propiedades de las láminas de grafeno dopado frente a la adsorción de oxígeno.

\section{MATERIALES Y MÉTODOS}

Se emplean métodos de cálculo basados en la Teoría del Funcional de la Densidad. La misma está fundada en los teoremas de Hohenberg y Kohn, [11] y es de gran utilidad en el estudio de la reactividad de sistemas químicos a nivel atómico y molecular.

La minimización de la ecuación para la energía considerando fijo el número total de electrones permite establecer una ecuación de Euler-Lagrange, $[12,13]$ que permite calcular el indicador de reactividad conocido como potencial químico, identificado también como el negativo de la electronegatividad., y que está relacionado con la tendencia de escape de los electrones desde una distribución de equilibrio, [13,14]. relación:

Una segunda función respuesta de la energía conduce a determinar la dureza química a través de la

$$
\eta=\left(\frac{\partial^{2} E}{\partial N^{2}}\right)_{v(r)}=\left(\frac{\partial \mu}{\partial N}\right)_{v(r)}
$$

Esta cantidad se interpreta como la resistencia de un sistema a trasferir carga y depende de $N$ y $v(r)$ y cumple un papel relevante en la estabilidad y reactividad química, [13]: una mayor estabilidad está asociada a una dureza máxima tal como establece el principio de máxima dureza.

Se puede obtener estos dos indicadores considerando que, para una molécula de capa cerrada, cada energía de un orbital ocupado es una buena aproximación del negativo de la energía necesaria para arrancar un electrón de ese orbital (energía de ionización). Esto es así, en DFT, para el orbital más alto ocupado (HOMO, Highest Occupied Molecular Orbital), que es igual al negativo de la energía de ionización molecular. De modo similar, la afinidad electrónica está dada por la energía del primer orbital desocupado (LUMO, Lowest Unoccupied Molecular Orbital), de la especie neutra. Entonces, las ecuaciones para $\eta$ se expresa como:

$$
\eta \cong \frac{1}{2}(P I-E A) \approx \frac{1}{2}\left(\varepsilon_{L}-\varepsilon_{H}\right)
$$

Cuando la dureza química es grande, los sistemas son más estables y pocos reactivos, como es el caso de los sistemas de capa cerrada. En sistemas de capa abierta, esta diferencia de energía es pequeña lo que indica que el sistema es poco estable y altamente reactivo.

Otra cantidad relacionada con la dureza es la blandura molecular que se determina utilizando la expresión:

$$
S=\frac{1}{2 \eta}=\frac{1}{\left(\varepsilon_{L}-\varepsilon_{H}\right)}
$$

Los términos de dureza y blandura adquirieron significado luego del trabajo realizado por Pearson, [15]. En este sentido se considera la siguiente distinción entre especies químicas: base blanda: especie donadora de electrones con alta polarizabilidad y baja electronegatividad (alto potencial químico), fácilmente oxidable. Se asocia con HOMO de alta energía; base dura: especie donadora de electrones con baja polarizabilidad y alta electronegatividad, difícil de oxidar; ácido blando: especie aceptora de electrones con pequeña carga positiva, sus electrones externos son fácilmente excitables, LUMO bajo; ácido duro: especie aceptora de electrones cuyos electrones externos no son fácilmente exitables.

Se analizan, además, otras cantidades de interés, el momento dipolar y la polarizabilidad [16]. El momento dipolar es una medida de la polaridad de la molécula; surge cuando hay una separación de carga, 
resultado de las diferencias en electronegatividad entre los átomos. Cuanto mayor sea la diferencia en electronegatividad, mayor es el momento dipolar. La distancia entre la separación de carga es también un factor decisivo en el valor del momento dipolar. El momento dipolar estático se expresa en función de sus componentes como:

$$
\mu=\left(\mu_{x}^{2}+\mu_{y}^{2} \mu_{z}^{2}\right)^{\frac{1}{2}}
$$

La polarizabilidad es una propiedad que se relaciona conceptualmente con el modo en que un campo eléctrico externo modifica la densidad electrónica del sistema en estudio. Formalmente es un tensor que se evalúa mediante la segunda derivada de la densidad respecto a un campo eléctrico externo y el campo eléctrico generado por los núcleos. Los elementos de la diagonal de ese tensor permiten determinar la polarizabilidad estática:

$$
\alpha_{0}=\frac{1}{3}\left(\alpha_{x x}+\alpha_{y y}+\alpha_{z z}\right)
$$

La polarizabilidad estática $\alpha_{0}$ es proporcional al número de electrones del sistema y es muy sensible a la deslocalización de electrones de valencia como así también a la forma y estructura del sistema.

La polarizabilidad total se determina mediante la expresión:

$$
\Delta \alpha=\left[\frac{\left(\alpha_{x x}-\alpha_{y y}\right)^{2}+\left(\alpha_{y y}-\alpha_{z z}\right)^{2}+\left(\alpha_{z z}-\alpha_{x x}\right)^{2}}{2}\right]^{\frac{1}{2}}
$$

La aplicación de la Teoría del Funcional de la Densidad a los sistemas en estudio, se realizó según el código del Programa Gaussian09 [17]. Se adoptó el funcional de Perdew-Burke-Ernzerhof, 1996 + HartreeFock intercambio, Perdew-Burke-Ernzerhof 1996 no local (1.0) + Perdew-Wang 1991, (PBE1PBE) y los pseudopotenciales de núcleo efectivo (LANL2DZ) para los átomos metálicos y las bases de George Petersson y colaboradores $(6-31 \mathrm{G}++(\mathrm{d}))$ para los átomos de carbono y oxígeno.

La lámina de grafeno fue modelada con 42 átomos de carbono. El dopado con átomos metálicos consistió en sustituir el átomo de carbono del centro de la lámina por un átomo metálico de $\mathrm{Fe}, \mathrm{Co}, \mathrm{y} \mathrm{Ni}$, respectivamente, como se muestra en la Figura 1.

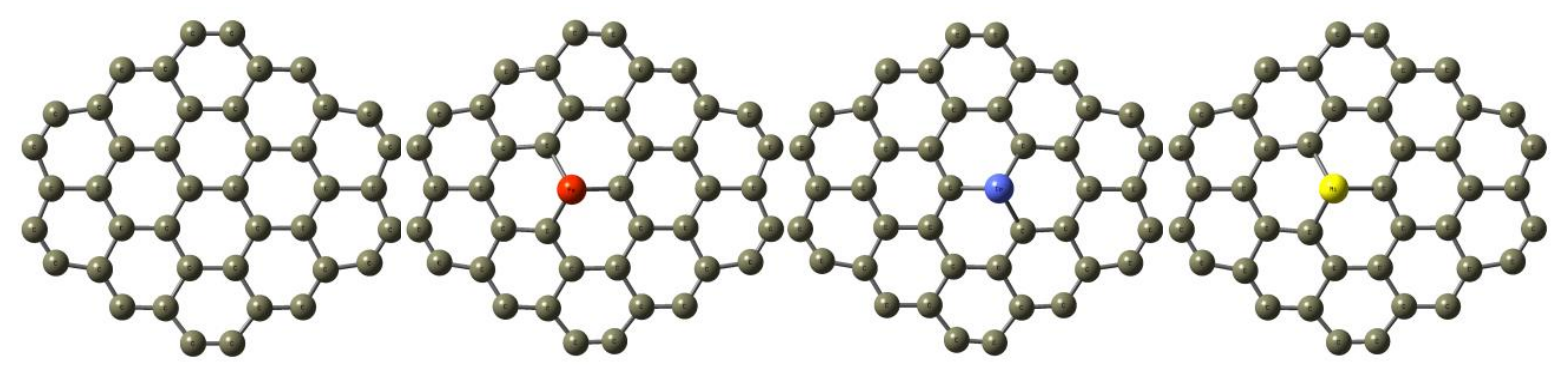

Figura 1: Modelado de la lámina de grafeno puro $C_{42}$ y dopada con átomos metálicos. $C_{41} F e, C_{41} \mathrm{Co}$ y $C_{41} N i$, respectivamente.

Para determinar los efectos del solvente se empleó el Modelo Polarizable Continuo (PCM) en el cual el valor de la energía de solvatación $\left(\Delta G_{\text {solv }}\right)$. Se expresa como:

$$
\Delta G_{\text {solv }}=\Delta G_{e l}+\Delta G_{c a v}+\Delta G_{r e p}+\Delta G_{d i s}+\Delta G_{t m}
$$

donde $\Delta G_{e l}$ es la diferencia entre la energía electrónica del soluto en fase gaseosa y en solución; $\Delta G_{r e p}$ es la contribución esférica o energía de repulsión, la que se calcula simulando una distribución de cargas en la superficie externa; $\Delta G_{\text {dis }}$ es la energía dispersiva, la cual se obtiene utilizando integrales bielectrónicas en el espacio de orbitales moleculares; finalmente, $\Delta G_{t m}$ es la energía térmica del movimiento nuclear. 


\section{RESULTADOS Y DISCUSIÓN}

\subsection{Análisis energético y electrónico}

Los orbitales de frontera, el orbital molecular ocupado más alto (HOMO) y el orbital molecular desocupado más bajo (LUMO) son factores importantes, ya que determinan la forma en que el sistema interactúa con otras especies. La diferencia de energía de frontera (gap de energía) ayuda a caracterizar la reactividad química y la estabilidad cinética del sistema. Un sistema con un gap pequeño es más polarizable y generalmente está asociado con una alta reactividad química, baja estabilidad cinética y también se denomina sistema blando.

Los sistemas propuestos para este estudio, grafeno puro $\left(\mathrm{C}_{42}\right)$ y dopado $\left(\mathrm{C}_{41} \mathrm{X}, \mathrm{X}=\mathrm{Fe}, \mathrm{Co}, \mathrm{Ni}\right)$, se optimizan hasta encontrar sus geometrías de mínima energía. Una vez conseguida la estabilidad termodinámica de esas estructuras se determina la energía de unión por átomo $\left(E_{u / a t}\right)$ por medio de la expresión:

$$
E_{u / a t}=\frac{\left(E_{C 41 X}-41 E_{C}-E_{X}\right)}{42}
$$

donde $E_{C 41 x}, E_{C}$ y $E_{X}$ son las energías de grafeno dopado, del átomo de carbono y del átomo dopante (en este caso $\mathrm{Fe}, \mathrm{Co} \mathrm{y} \mathrm{Ni)}$, respectivamente. Para el caso de grafeno puro se utilizó una expresión idéntica sin tener en cuenta la presencia de impurezas. En la Tabla 1 se presentan los resultados en vacío y con solvente.

Tabla 1: Energía de unión por átomo ( $\left.E_{u / a t}\right)$ para los sistemas $\mathrm{C}_{41} \mathrm{X}(\mathrm{X}=\mathrm{Fe}, \mathrm{Co}, \mathrm{Ni})$ en vacío y en solvente, $H_{2} O$. Energía de los orbitales de frontera $\operatorname{HOMO}\left(E_{H},\right)$ y LUMO $\left(E_{L}\right)$, gap de energía $(\Delta E)$ y Dureza, $(\eta)$. Los valores se expresan en $\mathrm{eV}$. Blandura, $(S)$, en $\mathrm{eV}^{-1}$.

\begin{tabular}{c|l|l|l|l|l|l|l|l}
\hline Sistema & $\mathrm{C}_{42}$ & $\mathrm{C}_{42} \mathrm{H}_{2} \mathrm{O}$ & $\mathrm{C}_{41} \mathrm{Fe}$ & $\mathrm{C}_{41} \mathrm{Fe} \mathrm{H}_{2} \mathrm{O}$ & $\mathrm{C}_{41} \mathrm{Co}$ & $\mathrm{C}_{41} \mathrm{Co} \mathrm{H}_{2} \mathrm{O}$ & $\mathrm{C}_{41} \mathrm{Ni}$ & $\mathrm{C}_{41} \mathrm{Ni} \mathrm{H}_{2} \mathrm{O}$ \\
\hline $\mathrm{E}_{\mathrm{u} / \mathrm{at}}$ & 7,85 & 7,50 & 7,63 & 7,42 & 7,64 & 7,42 & 7,60 & 7,26 \\
\hline $\mathrm{E}_{\mathrm{L}}$ & $-5,21$ & $-5,15$ & $-5,09$ & $-4,85$ & $-3,97$ & $-3,74$ & $-5,34$ & $-5,13$ \\
\hline $\mathrm{E}_{\mathrm{H}}$ & $-5,79$ & $-5,72$ & $-5,64$ & $-5,40$ & $-5,88$ & $-4,66$ & $-5,76$ & $-5,55$ \\
\hline$\Delta \mathrm{E}$ & 0,58 & 0,57 & 0,55 & 0,55 & 1,91 & 0,92 & 0,42 & 0,42 \\
\hline$\eta$ & 0,290 & 0,285 & 0,285 & 0,275 & 0,955 & 0,460 & 0,210 & 0,210 \\
\hline
\end{tabular}

En la Tabla 1 se observa que el grafeno, en vacío, es el sistema que presenta mayor estabilidad energética con un valor de energía de unión por átomo de 7,85 eV. La presencia del solvente provoca una disminución en la estabilidad energética respecto al grafeno puro. El sistema dopado con níquel, con un valor de 7,60 eV, es el que presenta una menor estabilidad.

La determinación del gap de energía $(\Delta \mathrm{E})$, que proviene de la diferencia entre los orbitales frontera HOMO $\left(E_{H}\right)$ y LUMO $\left(E_{L}\right)$, caracteriza la estabilidad electrónica. Un valor menor de gap caracteriza sistemas más reactivos, mientras que un mayor gap indica sistemas más estables. En la Figura 2 se observa que en el vacío, el mayor gap de energía corresponde al sistema dopado con cobalto $\left(C_{41} C o\right)$, con un valor de $1,91 \mathrm{eV}$, y el menor valor lo presenta el sistema dopado con níquel $\left(C_{41} \mathrm{Ni}\right)$ con $0,42 \mathrm{eV}$. La presencia del solvente modifica considerablemente el gap de energía del sistema $C_{41} C o$ disminuyendo $0,91 \mathrm{eV}$ mientras que para los sistemas dopados con hierro y níquel no se produce ninguna modificación y para el grafeno puro la disminución es muy pequeña. En consecuencia, el sistema más estable en el vacío es el dopado con cobalto y la presencia de solvente provoca una disminución del gap de energía, otorgándole una mayor reactividad.

En cuanto a la dureza química $\eta$, se observa que la estructura más dura o menos reactiva es la $C_{41} C o$ con un valor de $0,96 \mathrm{eV}$, en coincidencia con el mayor gap de energía encontrado para esta estructura. La presencia del solvente disminuye notablemente la dureza química del sistema $C_{41} \mathrm{Co}$ ya que presenta un valor $0,5 \mathrm{eV}$ menor respecto al sistema en el vacío; sin embargo, sigue siendo el sistema más duro o menos reactivo de la serie. En los otros sistemas el solvente no modifica la dureza química. El menor valor de dureza lo presenta el sistema $\mathrm{C}_{41} \mathrm{Ni}$ y ese valor se mantiene ante la presencia de solvente. 


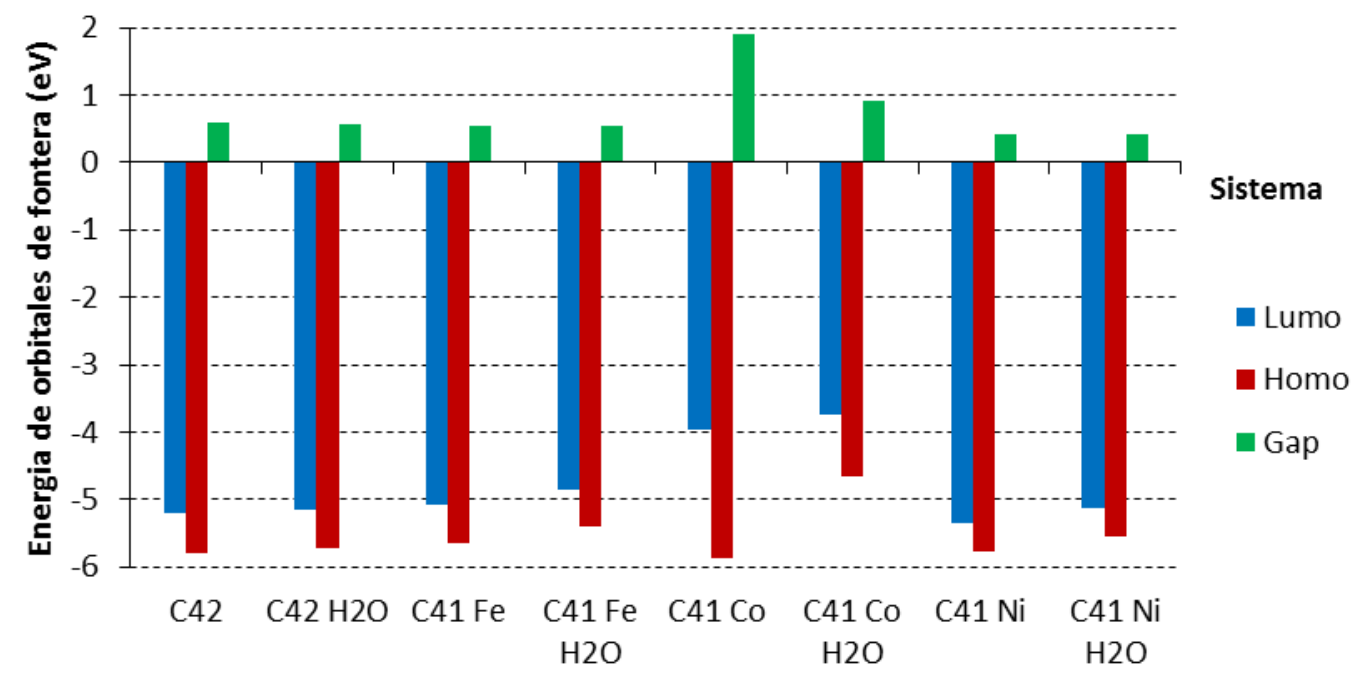

Figura 2: Energía de los orbitales de frontera para los sistemas en estudio, en vacío y en solvente (agua). Valores expresados en $\mathrm{eV}$.

\subsection{Momento dipolar, polarizabilidad estática y polarizabilidad total}

En la Tabla 2 se presentan los resultados obtenidos para el momento dipolar y el tensor de polarizabilidad, con sus respectivas componentes. Se registran los valores en vacío y en solvente.

Tabla 2: Momento dipolar total, $\mu$ (Debye) y polarizabilidad promedio, $\alpha_{0}$ (u.a.), y polarizabilidad total $\Delta \alpha$ (u.a.), para los sistemas en estudio.

\begin{tabular}{c|l|l|l|l|l|l|l|l}
\hline Sistema & \multicolumn{1}{|c|}{$\mathrm{C}_{42}$} & $\mathrm{C}_{41} \mathrm{H}_{2} \mathrm{O}$ & $\mathrm{C}_{41} \mathrm{Fe}$ & $\mathrm{C}_{41} \mathrm{Fe} \mathrm{H}_{2} \mathrm{O}$ & $\mathrm{C}_{41} \mathrm{Co}$ & $\mathrm{C}_{41} \mathrm{Co}_{2} \mathrm{O}$ & $\mathrm{C}_{41} \mathrm{Ni}$ & $\mathrm{C}_{41} \mathrm{Ni} \mathrm{H}_{2} \mathrm{O}$ \\
\hline$\mu_{x}$ & 0,0000 & $-0,0001$ & $-1,7221$ & $-4,8990$ & $-0,1694$ & 0,5744 & 0,1664 & $-1,9937$ \\
\hline$\mu_{y}$ & 0,0000 & 0,0000 & 0,3557 & 1,0930 & $-0,0001$ & 0,1128 & 0,0003 & 0,0123 \\
\hline$\mu_{z}$ & 1,0691 & 1,3831 & 2,4076 & 5,0227 & 1,6148 & 4,4577 & 2,0746 & 5,6474 \\
\hline$\mu$ & 1,0691 & 1,3831 & 2,9814 & 7,1009 & 1,6236 & 4,4959 & 2,0812 & 5,9889 \\
\hline$\alpha_{x x}$ & 925,67 & 2013,86 & 930,70 & 1990,84 & 772,87 & 1316,75 & 927,34 & 2018,66 \\
\hline$\alpha_{x y}$ & 0,0009 & 0,0893 & 4,14 & 22,78 & 0,0012 & $-7,21$ & 0,0063 & 0,1657 \\
\hline$\alpha_{x z}$ & 775,44 & 1734,12 & 749,05 & 1591,69 & 602,93 & 1026,58 & 824,89 & 1974,59 \\
\hline$\alpha_{y y}$ & 0,0036 & 0,0066 & 5,65 & 11,52 & 0,414 & 0,1044 & 7,39 & 14,54 \\
\hline$\alpha_{y z}$ & 0,0011 & $-0,0019$ & $-9,51$ & $-12,99$ & 0,0021 & $-4,36$ & 0,047 & 0,1024 \\
\hline$\alpha_{z z}$ & 114,84 & 149,83 & 142,58 & 192,09 & 143,42 & 190,64 & 141,61 & 189,46 \\
\hline$\alpha_{0}$ & 605,32 & 1299,27 & 607,44 & 1258,21 & 506,41 & 844,66 & 631,28 & 1394,24 \\
\hline$\Delta \alpha$ & 873,93 & 1943,28 & 864,75 & 1895,50 & 711,81 & 1232,47 & 860,72 & 1922,64 \\
\hline
\end{tabular}

En la Tabla 2 se observa que para el caso de la lámina de grafeno puro la presencia del solvente no modifica demasiado el valor del momento dipolar. Para el caso de la lámina de grafeno dopada, la presencia de heteroátomos incrementa el momento dipolar. La lámina dopada con átomos de hierro y níquel son las que presentan una mayor variación de esta cantidad por la presencia del solvente $(4,1$ D y 3,9 D, respectivamente); esto nos indica que los sistemas incrementan su electronegatividad. 


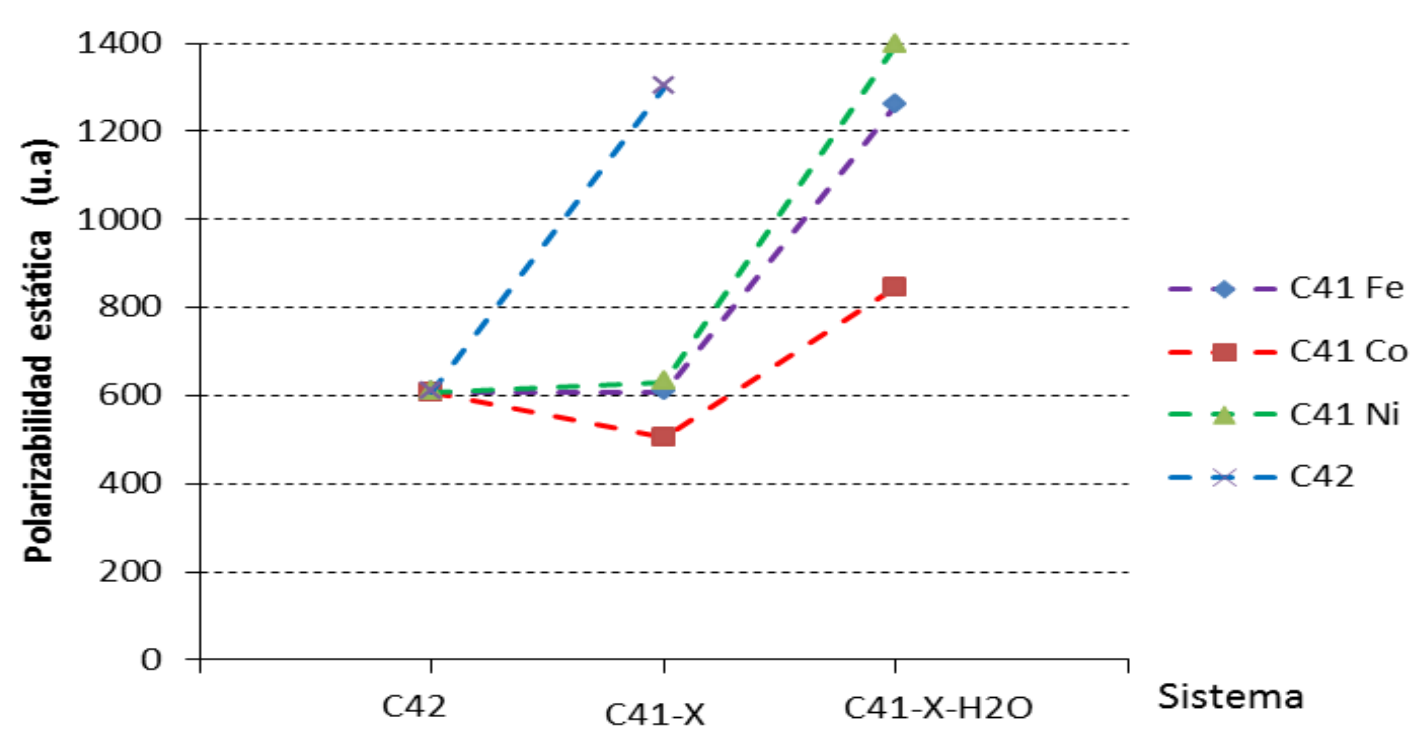

Figura 3: Comparación de la polarizabilidad estática para los sistemas en estudio con respecto a la del grafeno puro.

En la Figura 3 se observa la variación de la polarizabilidad estática de los sistemas dopados con respecto a la del grafeno puro. El sistema dopado con níquel es el que presenta una mayor variación de esta cantidad ante la presencia de solvente, de 631,28 u. a a 1394,24 u.a. Este sistema es, por lo tanto, el más sensible a la deslocalización de los electrones de valencia.

\subsection{Adsorción de oxígeno}

La energía de adsorción se determinó utilizando la expresión:

$$
E_{a d}=E_{c_{41} X O_{2}}-E_{C_{41} X}-E_{O_{2}}
$$

con $X \equiv \mathrm{Fe}$, Co y $\mathrm{Ni}$. Un valor negativo de $E_{a d}$ significa la adsorción favorable de $\mathrm{O}_{2}$ y un valor positivo significa la desorción del mismo. Los resultados se muestran en la Tabla 3.

Se observa que el sistema dopado que presenta mayor energía de adsorción es el sistema $C_{41} C o$ y el sistema que presenta menor energía de adsorción es $C_{41} \mathrm{Ni}$. La incidencia del solvente en todos los sistemas se analizó determinando la energía de solvatación. Esta energía es una medida de la interacción entre el soluto y el solvente en relación con la estabilización de las especies del soluto en la solución y se calculó realizando la diferencia entre la energía libre del sistema en solución y la energía del sistema en vacío. Los valores obtenidos se presentan en la Tabla 3.

Tabla 3: Energía de adsorción del oxígeno molecular sobre grafeno dopado $\mathrm{C}_{41} \mathrm{X}(\mathrm{X}=\mathrm{Fe}, \mathrm{Co}, \mathrm{Ni})$., $E_{a d}$ y energía de solvatación, $E_{\text {solv }}$.

\begin{tabular}{c|c|c|c}
\hline \multirow{2}{*}{ Sistema } & \multicolumn{2}{|c|}{$E_{a d}(\mathrm{eV})$} & \multirow{2}{*}{$E_{\text {solv }}(\mathrm{eV})$} \\
\cline { 2 - 3 } & En Vacío & En Solvente & \\
\hline$C_{41} \mathrm{FeO}_{2}$ & $-2,12$ & $-1,83$ & $-0,19$ \\
\hline$C_{41} \mathrm{CoO}_{2}$ & $-2,83$ & $-2,84$ & $-0,15$ \\
\hline$C_{41} \mathrm{NiO}_{2}$ & $-1,38$ & $-0,87$ & $-0,23$ \\
\hline
\end{tabular}

\section{CONCLUSIONES}

Mediante el empleo de cálculos DFT se han estudiado las propiedades electrónicas y energéticas de una lámina de grafeno dopada con átomos metálicos $3 \mathrm{~d}$ (Fe, Co, Ni). Se utilizó el modelo PCM para considerar la incidencia del solvente. De los resultados podemos arribar a las siguientes conclusiones:

- El análisis energético y electrónico mediante indicadores globales de reactividad nos permite con- 
cluir que el sistema más reactivo en el $C_{41} N i$ y el menos reactivo o más estable es el sistema $C_{41} C o$.

- El momento dipolar y la polarizabilidad se incrementan con la presencia de heteroátomos y la presencia del solvente.

- La presencia heteroátomos y del solvente, en todos los casos, promueve un debilitamiento en la energía de adsorción de oxígeno, siendo el sistema dopado con níquel el que presenta el mayor debilitamiento por lo que facilitaría la disociación de oxígeno.

- Los resultados obtenidos son un punto de partida interesante para el estudio de las propiedades catalíticas de estos sistemas frente a la reducción de oxígeno, mediante cálculos del camino de reacción y determinación de estados de transición.

\section{BIBLIOGRAFÍA}

[1] TOH, R. J., POH, H.L., SOFER, Z.K., et al., "Transition Metal (Mn, Fe, Co, Ni)-Doped Graphene Hybrids for Electrocatalysis", Chemistry Asian Journal, v.8, n.6, pp.1295 - 1300, 2013.

[2] ZHANG, L., NIU, J., LI, M., et al., "Catalytic Mechanisms of Sulfur-Doped Graphene as Efficient Oxygen Reduction Reaction Catalysts for Fuel Cells", The Journal Physical Chemistry, v.118, pp. 35453553, 2014.

[3] KAUKONEN, M., KRASHENINNIKOV, A. V., KAUPPINEN, E., et al., "Doped Graphene as a Material for Oxygen Reduction Reaction in Hydrogen Fuel Cells: A Computational Study", American Chemical Society, v.3, n.2, pp. 159-165, marzo de 2013.

[4] TABTIMSAI, C., TONTAPHA, S., RAKRAI, W., et al., "DFT study on structural stability and electronic property of VIIIB transition metal-doped carbon nanocaps", Solid State Sciences, v.37, julio 2014.

[5] KUILA, T., BOSE, S., MISHRA, A.K., et al., "Chemical functionalization of graphene and its applications", Progress in Materials Science, v.57, n.7, pp.1061-1105, Marzo de 2012.

[6] NASEHNIA, F., SEIFI, M, "Adsorption of molecular oxygen on VIIIB transition metal-doped graphene: A DFT study", Modern Physics Letters B, v. 28, n. 30 1450237. Diciembre de 2014.

[7] GIOVANNI, M., POH, H.L., AMBROSI, A., et al., "Noble metal (Pd, Ru, Rh, Pt, Au, Ag) doped graphene hybrids for electrocatalysis", Nanoscale, v.4, n.16, pp.5002-5008, junio de 2012.

[8] LI, Y, FAN, X., QI, J., et al., "Palladium nanoparticle-graphene hybrids as active catalysts for the Suzuki reaction", Nano Research, v.3, n.6, pp.429-437, junio de 2010.

[9] TOH, R.J., POH, H.L, SOFER, Z.K., et al., "Transition Metal (Mn, Fe, Co, Ni)-Doped Graphene Hybrids for Electrocatalysis", Chem. Asian J., v. 8, pp. 1295 - 1300, Junio de 2013.

[10] CARLTON, C., CHEN, S., FERREIRA, P., et al., "Sub-Nanometer-Resolution elemental mapping of Pt3Co nanoparticle catalyst degradation in proton-exchange membrane fuel cells", The Journal of Physical Chemistry Letters, pp 161-166, Marzo de 2012.

[11] HOHENBERG, P., KOHN, W. "Inhomogeneous electron gas", Physics. Review B, v. 136, pp. 864-971, noviembre de 1964.

[12] PARR, R. G., DONNELLY, R. A., LEVY, M., et al., "Electronegativity: The density functional viewpoint", Journal Physical Chemistry, v. 68, pp. 3801-3807, Abril de 1978.

[13] CHERMETTE, H. "Chemical reactivity indexes in density functional theory", Computational Chemistry, v. 20, pp. 129-154, Enero de 1999.

[14] GEELINGS, P., PROFT, F., LANGENAEKER, W., "Conceptual density functional theory", Chemical Review, v. 103, pp. 1793-1873. Mayo de 2003.

[15] NALEWAJSKI, R. F. "Electronic structure and chemical reactivity: density functional and informationtheoretic perspectives", Advances Quantum Chemistry, v. 43, pp. 119-184, 2003.

[16] KERASSA, A., BELAIDI,S., DALAL, H., et al., "Investigations on Molecular Structure, Electronic Properties, NLO Properties, HOMO-LUMO Analysis and Comparison of Drug-likeness of Triazolothiadiazole Derivatives by Quantum methods and QSAR Analysis", Reviews in Theoretical Science, v. 3, pp. 1-10, Marzo 2015.

[17] FRISCH, M.J., TRUCKS, G. W., SCHLEGEL, H. B., et al., "Gaussian 09, revision”, Gaussian, Inc., Wallingford C. T., 2009. 